Research Article

\title{
Stability Analysis and Control of a New Smooth Chua's System
}

\author{
Guopeng Zhou, ${ }^{1,2}$ Jinhua Huang, ${ }^{3}$ Xiaoxin Liao, ${ }^{4}$ and Shijie Cheng ${ }^{1}$ \\ ${ }^{1}$ College of Electrical and Electronic Engineering, Huazhong University of Science and Technology, \\ Wuhan 430074, China \\ ${ }^{2}$ College of Electronic and Information Engineering, Hubei University of Science and Technology, \\ Xianning 437100, China \\ ${ }^{3}$ Department of Electric and Electronic Engineering, Wuhan Institute of Shipbuilding Technology, \\ Wuhan 430050, China \\ ${ }^{4}$ Department of Control and Engineering, Huazhong University of Science and Technology, \\ Wuhan 430074, China
}

Correspondence should be addressed to Jinhua Huang; angela_icec@yahoo.com.cn

Received 3 December 2012; Accepted 7 April 2013

Academic Editor: René Yamapi

Copyright (C) 2013 Guopeng Zhou et al. This is an open access article distributed under the Creative Commons Attribution License, which permits unrestricted use, distribution, and reproduction in any medium, provided the original work is properly cited.

This paper is concerned with the stability analysis and control of a new smooth Chua's system. Firstly, the chaotic characteristic of the system is confirmed with the aid of the Lyapunov exponents. Secondly, it is proved that the system has globally exponential attractive set and positive invariant set. For the three unstable equilibrium points of the system, a linear controller is designed to globally exponentially stabilize the equilibrium points. Then, a linear controller and an adaptive controller are, respectively, proposed so that two similar types of smooth Chua's systems are globally synchronized, and the estimation errors of the uncertain parameters converge to zero as $t$ tends to infinity. Finally, the numerical simulations are also presented.

\section{Introduction}

It is well known that Chua's system is the first analog circuit to realize chaos in experiments. The original Chua's system is described by the following ordinary differential equations [1]:

$$
\begin{gathered}
\dot{x}=p(y-x-g(x)), \\
\dot{y}=x-y+z, \\
\dot{z}=-q y,
\end{gathered}
$$

where $x, y, z \in R$ are state variables and $g(x)=G_{b} x+$ $(1 / 2)\left(G_{a}-G_{b}\right)(|x-E|-|x-E|), p>0, q>0$, and $G_{a}, G_{b}$ are constants. Due to the form of a simple circuit, there are a large literature on the dynamical behavior of Chua's system [2-8]. By changing the parameters or the corresponding functions of Chua's system, the chaotic phenomenon is very rich, and it is more convenient to study the chaotic mechanism and characteristics [6-8].

For the chaotic systems, Lagrange stability, stability of equilibrium points and, synchronization are three important problems which attracted more and more attention (refer to [9-16] and the reference therein). In [11-13], the authors studied the Lagrange stability by applying the attractive set and positive invariant set of the chaotic systems. Moreover, the researchers examined the stabilization of the unstable equilibrium points and the synchronization control for the chaotic systems with linear controllers $[7,14]$. Recently, adaptive controllers are used in synchronous control of chaotic systems when the parameters of the systems are uncertain [15-17].

Motivated by the previous results, the main purpose of this paper is to construct a new smooth Chua's system and 


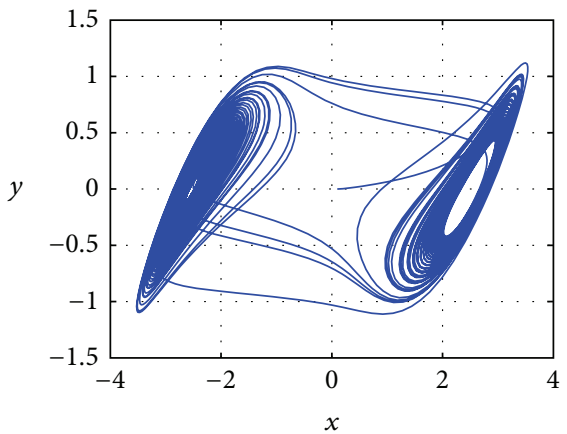

(a)

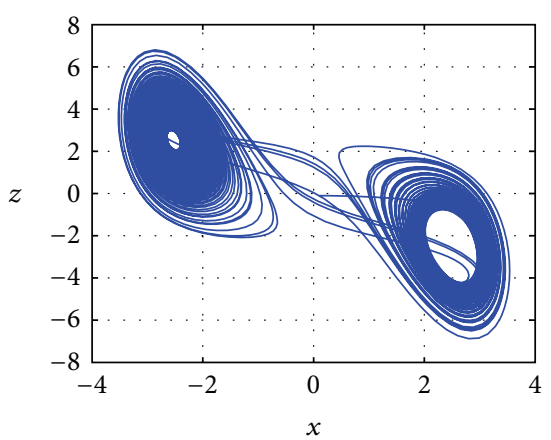

(b)

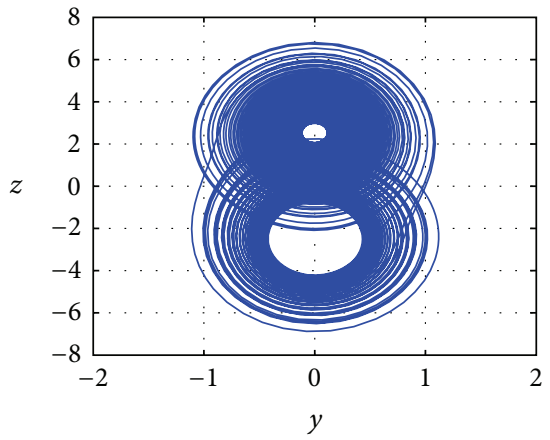

(c)

FIgURE 1: The phase portraits of Chua's system (2).

investigate the stability and control problems. More precisely, we will consider the following smooth Chua's system:

$$
\begin{gathered}
\dot{x}=p\left(x+y-x \ln \sqrt{1+x^{2}}\right), \\
\dot{y}=x-y+z, \\
\dot{z}=-q y,
\end{gathered}
$$

where $x, y, z \in R$ are state variables and $p>0, q>0$ are constants.

We will show that the chaotic characteristics are depended on the parameters $p$ and $q$ and the initial state values of the system (2). All equilibrium points of system (2) are examined to be unstable when $p=11$ and $q=14.87$ (see in Section 3). By computing with MATLAB, the maximum Lyapunov exponent of the system (2) is 0.0021 , where the embedding dimension $m$ is 3 and the delay time $\tau$ is 5 . Since the maximum Lyapunov exponent is greater than 0 , the Chua's system is chaotic. It will be of great significance if the solution of (2) is ultimately bounded (Lagrange asymptotically stable). The chaotic phase diagrams of such system is obtained by simulation with MATLAB. Figure 1 shows the phase diagrams of the system (2) with $x(0)=-3$, $y(0)=2$, and $z(0)=1$, the phase diagrams of Chua's system exhibits chaotic.

The remains of this paper are organized as follows. The existence of globally exponential attractive set and positive invariant set for the system (2) will be discussed in Section 2. The asymptotic stability of the equilibrium points will be studied in Section 3, and the synchronization control for two similar types of the Chua's systems will be discussed in Section 4. In Section 5, we will give the numerical simulations to demonstrate the correctness of our results, and finally we will give the conclusions in Section 6.

\section{Existence of Globally Exponential Attractive Set and Positive Invariant Set}

The Lagrange stability analysis of the system (2) will be studied in this section. To do so, we first give two definitions [7].
Definition 1. If there exists a radially unbounded, positive definite Lyapunov function $V(X(t))$ and positive numbers $l>0, \alpha>0$ such that for all $X_{0} \in R^{3}$, when $V\left(X_{0}\right)>l$, the solution of the system (2), $X(t)=(x(t), y(t), z(t))$ along $V(X(t))$ satisfies $|V(X(t))-l| \leq\left|V\left(x_{0}\right)-l\right| e^{-\alpha\left(t-t_{0}\right)}$, then system (2) has a globally exponential attractive set $\Omega \triangleq\{X \mid$ $V(X(t))<l\}$.

Definition 2. Let $\Omega \subseteq R^{3}$, if $\forall X_{0} \in \Omega$ and for all $t \geq t_{0}$, $X\left(t, t_{0}, X_{0}\right) \subseteq \Omega$, then $\Omega$ is called positive invariant set of the system (2).

It is easy to prove that the globally exponential attractive set is positive invariant. A system with global attractive set is always called Lagrange globally asymptotically stable system or ultimately bounded dissipative system. For the system (2), we will prove the following the Lagrange stability results.

Theorem 3. The system (2) has the following globally exponential attractive and positive invariant set:

$$
\Omega=\left\{\begin{array}{l}
|x| \leq\left|x_{b}\right|=\min \left\{\left|x_{b 1}\right|,\left|x_{b 2}\right|\right\} \\
y^{2}+\frac{1}{q} z^{2}-\epsilon y z \leq \frac{(5 / 4 \eta) x_{b}^{2}}{-\tau_{1} \mu_{M}\left(G_{2}^{-}\right) / \lambda_{M}\left(G_{2}^{+}\right)} \\
\left|x_{b 1}\right|>\sqrt{e^{\left((2-(\epsilon / 4))^{2} /(2-\epsilon q-(\epsilon / 4))\right)+(\epsilon / 4)+2}-1} \\
\left|x_{b 2}\right|=\sup _{\bar{\Omega}}|x|, \\
\bar{\Omega} \triangleq\left\{x \mid \frac{1}{p} x^{2}+y^{2}+\frac{1}{q} z^{2}-\epsilon y z\right. \\
\left.=\frac{x_{b 1}^{2} \ln \left(1+x_{b 1}^{2}\right)}{-\mu_{M}\left(G_{1}^{-}\left(x_{b 1}\right)\right) / \lambda_{M}\left(G_{1}^{+}\right)}\right\},
\end{array}\right.
$$


where $0<\epsilon \ll 1$,

$$
G_{1}^{+} \triangleq\left[\begin{array}{ccc}
\frac{1}{p} & 0 & 0 \\
0 & 1 & -\frac{\epsilon}{2} \\
0 & -\frac{\epsilon}{2} & \frac{1}{q}
\end{array}\right], \quad G_{2}^{+} \triangleq\left[\begin{array}{cc}
1 & -\frac{\epsilon}{2} \\
\epsilon & \frac{1}{2}
\end{array}\right],
$$

are symmetric positive definite matrices, and

$$
\begin{gathered}
G_{1}^{-} \triangleq\left[\begin{array}{crc}
2\left(1-\ln \sqrt{1+x_{b 1}^{2}}\right) & 2 & -\frac{\epsilon}{2} \\
2 & \epsilon q-2 & \frac{\epsilon}{2} \\
-\frac{\epsilon}{2} & \frac{\epsilon}{2} & -\epsilon
\end{array}\right], \\
G_{2}^{-} \triangleq\left[\begin{array}{cc}
\epsilon q-2 & \frac{\epsilon}{2} \\
\frac{\epsilon}{2} & -\epsilon
\end{array}\right]
\end{gathered}
$$

are symmetric negative definite matrices. $\lambda_{M}\left(G_{1}^{+}\right), \mu_{M}\left(G_{1}^{-}\right)$, $\lambda_{M}\left(G_{2}^{+}\right)$, and $\mu_{M}\left(G_{2}^{-}\right)$are the maximum eigenvalues of $G_{1}^{+}$, $G_{1}^{-}, G_{2}^{+}$, and $G_{2}^{-}$, respectively. $\epsilon, x_{b 1}$, and $x_{b 2}$ in (3) are chosen to guarantee that $G_{1}^{+}, G_{2}^{+}$are positive definite and $G_{1}^{-}, G_{2}^{-}$are negative definite. $\tau_{1}, \tau_{2}$, and $\eta$ are chosen such that $\tau_{1}>0$, $\tau_{2}>0, \eta>0, \tau_{1}+\tau_{2}=1$, and $\tau_{2} \mu_{M}\left(G_{2}^{-}\right)+\eta \leq 0$.

Proof. The proof is divided into three steps.

Step I. The existence of $\epsilon, x_{b 1}$ such that $G_{1}^{+}$is positive definite and $G_{1}^{-}$is negative definite.

It is well known that $G_{1}^{+}$is positive definite if and only if all the order principal minors of $G_{1}^{+}$are positive [18]. That is,

$$
\begin{gathered}
\frac{1}{p}>0, \quad\left|\begin{array}{ll}
\frac{1}{p} & 0 \\
0 & 1
\end{array}\right|>0, \\
\left|\begin{array}{ccc}
\frac{1}{p} & 0 & 0 \\
0 & 1 & -\frac{\epsilon}{2} \\
0 & -\frac{\epsilon}{2} & \frac{1}{q}
\end{array}\right|=\frac{1}{p}\left(\frac{1}{q}-\frac{\epsilon^{2}}{4}\right)>0 .
\end{gathered}
$$

Let $|\epsilon|<2 / \sqrt{q}$; it is easy to see that $G_{1}^{+}$is positive definite since $p>0, q>0$.

Next, let

$$
\begin{gathered}
S_{11}=\left[\begin{array}{cc}
2\left(1-\ln \sqrt{1+x_{b 1}^{2}}\right) & 2 \\
2 & \epsilon q-2
\end{array}\right], \\
S_{12}=\left[\begin{array}{c}
-\frac{\epsilon}{2} \\
\frac{\epsilon}{2}
\end{array}\right], \quad S_{21}=\left[-\frac{\epsilon}{2}, \frac{\epsilon}{2}\right], \quad S_{22}=-\epsilon .
\end{gathered}
$$

Then,

$$
G_{1}^{-}=\left[\begin{array}{ll}
S_{11} & S_{12} \\
S_{21} & S_{22}
\end{array}\right]
$$

From Schur theorem [18], $G_{1}^{-}$is negative definite if and only if $S_{11}<0, S_{22}-S_{21} S_{11}^{-1} S_{12}<0$. Let $|\epsilon|, x_{b 1}$ satisfy

$$
0<\epsilon \ll 1,
$$

$$
\left|x_{b 1}\right|>\sqrt{\mathrm{e}^{\left((2-(\epsilon / 4))^{2} /(2-\epsilon q-(\epsilon / 4))\right)+(\epsilon / 4)+2}-1} .
$$

It is easy to verify that $G_{1}^{-}$is negative definite.

Step II. Existence of globally exponential attractive set of $x$.

Let $\lambda_{m}\left(G_{1}^{+}\right)$and $\lambda_{m}\left(G_{1}^{-}\right)$be the minimum eigenvalues of $G_{1}^{+}$and $G_{1}^{-}$, respectively. By constructing a radially unbounded Lyapunov function as follows:

$$
V=\frac{1}{p} x^{2}+y^{2}+\frac{1}{q} z^{2}-\epsilon y z=\left[\begin{array}{c}
x \\
y \\
z
\end{array}\right]^{T} G_{1}^{+}\left[\begin{array}{l}
x \\
y \\
z
\end{array}\right],
$$

then, one can obtain that

$$
\lambda_{m}\left(G_{1}^{+}\right)\left(x^{2}+y^{2}+z^{2}\right) \leq V \leq \lambda_{M}\left(G_{1}^{+}\right)\left(x^{2}+y^{2}+z^{2}\right) .
$$

The time derivative of $V$ along the system (2) is given by

$$
\begin{aligned}
\left.\frac{d V}{d t}\right|_{(2)}= & \frac{2}{p} x \dot{x}+2 y \dot{y}+\frac{2}{q} z \dot{z}-\epsilon \dot{y} z-\epsilon y \dot{z} \\
= & \frac{2}{p} x p\left(x+y-x \ln \sqrt{1+x^{2}}\right)+2 y(x-y+z) \\
& +\frac{2}{q}(-q y)-\epsilon(x-y+z) z-\epsilon y(-q y) \\
= & {\left[\begin{array}{l}
x \\
y \\
z
\end{array}\right]^{T}\left[\begin{array}{ccc}
2\left(1-\ln \sqrt{1+x^{2}}\right) & 2 & -\frac{\epsilon}{2} \\
2 & \frac{\epsilon}{2} & -\epsilon
\end{array}\right]\left[\begin{array}{l}
x \\
y \\
z
\end{array}\right] } \\
= & {\left[\begin{array}{l}
x \\
y \\
z
\end{array}\right]^{T}\left[\begin{array}{ccc}
G_{1}^{-} \\
y \\
z
\end{array}\right]-2\left(\ln \sqrt{1+x^{2}}-\ln \sqrt{1+x_{b 1}^{2}}\right) x^{2} . }
\end{aligned}
$$

If $|x|>\left|x_{b 1}\right|$, one has

$$
\left.\frac{d V}{d t}\right|_{(2)} \leq\left[\begin{array}{l}
x \\
y \\
z
\end{array}\right]^{T} G_{1}^{-}\left[\begin{array}{l}
x \\
y \\
z
\end{array}\right] \leq \frac{\mu_{M}\left(G_{1}^{-}\right)}{\lambda_{M}\left(G_{1}^{+}\right)} V .
$$

Then, the following inequality holds

$$
V\left(X\left(t, t_{0}, X_{0}\right)\right) \leq V\left(X_{0}\right) \mathrm{e}^{\left(\mu_{M}\left(G_{1}^{-}\right) / \lambda_{M}\left(G_{1}^{+}\right)\right)\left(t-t_{0}\right)} .
$$


Thus, the trajectory of the system (2) will exponentially decay into the area $U=\left\{x|| x|\leq| x_{b 1} \mid\right\}$ if $|x|>\left|x_{b 1}\right|$.

When the trajectory of the system (2) is in the area $U$, it holds that

$$
\begin{aligned}
\left.\frac{d V}{d t}\right|_{(2)} & =\left[\begin{array}{l}
x \\
y \\
z
\end{array}\right]^{T} G_{1}^{-}\left[\begin{array}{l}
x \\
y \\
z
\end{array}\right]+2\left(\ln \sqrt{1+x_{b 1}^{2}}-\ln \sqrt{1+x^{2}}\right) x^{2} \\
& \leq \frac{\mu_{M}\left(G_{1}^{-}\right)}{\lambda_{M}\left(G_{1}^{+}\right)} V+2\left(\ln \sqrt{1+x_{b 1}^{2}}-\ln \sqrt{1+x^{2}}\right) x^{2} \\
& \leq \frac{\mu_{M}\left(G_{1}^{-}\right)}{\lambda_{M}\left(G_{1}^{+}\right)} V+x_{b 1}^{2} \ln \left(1+x_{b 1}^{2}\right) \\
& =\frac{\mu_{M}\left(G_{1}^{-}\right)}{\lambda_{M}\left(G_{1}^{+}\right)}\left(\frac{V-x_{b 1}^{2} \ln \left(1+x_{b 1}^{2}\right)}{-\mu_{M}\left(G_{1}^{-}\right) / \lambda_{M}\left(G_{1}^{+}\right)}\right) .
\end{aligned}
$$

Let $V_{l} \triangleq\left(x_{b 1}^{2} \ln \left(1+x_{b 1}^{2}\right)\right) /\left(-\mu_{M}\left(G_{1}^{-}\right) / \lambda_{M}\left(G_{1}^{+}\right)\right)$. If $V\left(X_{0}\right)>$ $V_{l}$ and $V\left(X\left(t, t_{0}, X_{0}\right)\right)>V_{l}$, it holds that

$$
V\left(X\left(t, t_{0}, X_{0}\right)\right)-V_{l} \leq\left(V\left(X_{0}\right)-V_{l}\right) e^{\left(\mu_{M}\left(G_{1}^{-}\right) / \lambda_{M}\left(G_{1}^{+}\right)\right)\left(t-t_{0}\right)} .
$$

Let $\bar{\Omega} \triangleq\left\{X \mid V(X)=V_{l}\right\},\left|x_{b 2}\right| \triangleq \sup _{x \in \bar{\Omega}}|x|$. Then, $V\left(X\left(t, t_{0}, X_{0}\right)\right)-V_{l}$ exponentially decays when $|x| \geq\left|x_{b}\right| \triangleq$ $\min \left\{\left|x_{b 1}\right|,\left|x_{b 2}\right|\right\}$. Thus, $\Omega_{x} \triangleq\left\{x|| x|\leq| x_{b} \mid\right\}$ is the globally exponential attractive set of $x$.

Step III. Existence of globally exponential attractive set of $y$ and $z$.

Let

$$
G_{2}^{+} \triangleq\left[\begin{array}{cc}
1 & -\frac{\epsilon}{2} \\
-\frac{\epsilon}{2} & \frac{1}{q}
\end{array}\right], \quad G_{2}^{-} \triangleq\left[\begin{array}{cc}
\epsilon q-2 & \frac{\epsilon}{2} \\
\frac{\epsilon}{2} & -\epsilon
\end{array}\right],
$$

then, $G_{2}^{+}$is a positive matrix if $\epsilon \in(0,2 / \sqrt{q})$, and $G_{2}^{-}$is a negative matrix if $\epsilon \in(0,8 /(4 q+1))$.

At the same time, it is easy to examine that $G_{2}^{+}$is positive and $G_{2}^{-}$is negative if let $\epsilon \in(0,8 /(4 q+1))$, since $q>0$ and $8 /(4 q+1)=2 /(q+1 / 4) \leq 2 / \sqrt{q}$.

Let $\lambda_{m}\left(G_{2}^{+}\right), \lambda_{M}\left(G_{2}^{+}\right)$be, respectively, minimum and maximum eigenvalues of $G_{2}^{+}$, and let $\mu_{m}\left(G_{2}^{-}\right)$and $\mu_{M}\left(G_{2}^{-}\right)$be the minimum and maximum eigenvalues of $G_{2}^{-}$, respectively. Since $|x| \leq\left|x_{b}\right|$, a radially unbounded and positive definite Lyapunov function about $y$ and $z$ is constructed as follows:

$$
W=y^{2}+\frac{1}{q} z^{2}-\epsilon y z=\left[\begin{array}{l}
y \\
z
\end{array}\right]^{T} G_{2}^{+}\left[\begin{array}{l}
y \\
z
\end{array}\right]
$$

Then, the time derivative of $W$ along the system (2) yields

$$
\begin{aligned}
\left.\frac{d W}{d t}\right|_{(2)}= & 2 y \dot{y}+\frac{2}{q} z \dot{z}-\epsilon \dot{y} z-\epsilon y \dot{z} \\
= & {\left[\begin{array}{c}
y \\
z
\end{array}\right]^{T} G_{2}^{-}\left[\begin{array}{l}
y \\
z
\end{array}\right]+2 x y-\epsilon x z } \\
\leq & \mu_{M}\left(G_{2}^{-}\right)\left(y^{2}+z^{2}\right)+2\left|x_{b}\right||y|+\epsilon\left|x_{b}\right||z| \\
\leq & \tau_{1} \mu_{M}\left(G_{2}^{-}\right)\left(y^{2}+z^{2}\right)+\tau_{2} \mu_{M}\left(G_{2}^{-}\right)\left(y^{2}+z^{2}\right) \\
& +\eta y^{2}+\frac{x_{b}^{2}}{\eta}+\eta z^{2}+\frac{x_{b}^{2}}{4 \eta} \\
\leq & \frac{\tau_{1} \mu_{M}\left(G_{2}^{-}\right)}{\lambda_{M}\left(G_{2}^{+}\right)} W+\left(\tau_{2} \mu_{M}\left(G_{2}^{-}\right)+\eta\right)\left(y^{2}+z^{2}\right)+\frac{5}{4 \eta} x_{b}^{2} \\
\leq & \frac{\tau_{1} \mu_{M}\left(G_{2}^{-}\right)}{\lambda_{M}\left(G_{2}^{+}\right)}\left(W-\frac{(5 / 4 \eta) x_{b}^{2}}{-\tau_{1} \mu_{M}\left(G_{2}^{-}\right) / \lambda_{M}\left(G_{2}^{+}\right)}\right)
\end{aligned}
$$

where $\tau_{1}, \tau_{2}$, and $\eta$ are chosen such that $\tau_{1}>0, \tau_{2}>0, \eta>0$, $\tau_{1}+\tau_{2}=1$, and $\tau_{2} \mu_{M}\left(G_{2}^{-}\right)+\eta \leq 0$.

Let $W_{l} \triangleq(5 / 4 \eta) x_{b}^{2} /\left(-\tau_{1} \mu_{M}\left(G_{2}^{-}\right) / \lambda_{M}\left(G_{2}^{+}\right)\right)$; if $W\left(X_{0}\right)>W_{l}$ and $W\left(X\left(t, t_{0}, X_{0}\right)\right)>W_{l}$, it holds that

$$
\begin{aligned}
& W\left(X\left(t, t_{0}, X_{0}\right)\right)-W_{l} \\
& \quad \leq\left(W\left(X_{0}\right)-W_{l}\right) e^{\left(\tau_{1} \mu_{M}\left(G_{2}^{-}\right) / \lambda_{M}\left(G_{2}^{+}\right)\right)\left(t-t_{0}\right)} .
\end{aligned}
$$

Similarly, if $W\left(X_{0}\right) \leq W_{l}$, the state trajectory will stay in the area such that $W\left(X\left(t, t_{0}, X_{0}\right)\right) \leq W_{l}$ holds. Hence, $y$ and $z$ are exponentially decreased and ultimately enter into the attractive region $W\left(X\left(t, t_{0}, X_{0}\right)\right) \leq W_{l}$, that is,

$$
y^{2}+\frac{1}{q} z^{2}-\epsilon y z \leq \frac{(5 / 4 \eta) x_{b}^{2}}{-\tau_{1} \mu_{M}\left(G_{2}^{-}\right) / \lambda_{M}\left(G_{2}^{+}\right)} .
$$

Combining Steps I, II, and III, Theorem 3 is obtained and the proof is completed.

Remark 4. In this section, a constructive method is proposed to prove the main results of existence of globally exponential attractive set and positive invariant set for the Chua's systems. By constructing the matrices $G_{1}^{+}, G_{1}^{-}, G_{2}^{+}, G_{2}^{-}$, and Lyapunov function candidate $V$ and $W$, the problem is solved ingeniously.

\section{Global Linear Stabilization of the Equilibrium Points}

In this section, the stability of the equilibrium points for the system (2) will be discussed with the aid of a linear controller.

Firstly, it is easy to examine that the system (2) has three equilibrium points:

$$
\begin{gathered}
S_{0}=(0,0,0), \quad S_{+}=\left(\sqrt{e^{2}-1}, 0,-\sqrt{e^{2}-1}\right), \\
S_{-}=\left(-\sqrt{e^{2}-1}, 0, \sqrt{e^{2}-1}\right) .
\end{gathered}
$$


Moreover, the equilibrium points of the system is independent of parameters $p$ and $q$. It should be mentioned that, however, the stability of the equilibrium points is depended on $p$ and $q$. In the following, we will design a linear controller to stabilize the unstable equilibrium points.

Let $p=11, q=14.87$, and $\left(x^{*}, y^{*}, z^{*}\right)$ be any equilibrium point of the system (2), the corresponding Jacobian matrix is given by

$$
J_{0}=\left[\begin{array}{ccc}
p\left(-\ln \sqrt{1+x^{* 2}}+\frac{1}{1+x^{* 2}}\right) & p & 0 \\
1 & -1 & 1 \\
0 & -q & 0
\end{array}\right]
$$

Then the characteristic equation of the corresponding local linearization system of system (2) is as follows:

$$
a_{0} \lambda^{3}+a_{1} \lambda^{2}+a_{2} \lambda+a_{3}=0
$$

where $a_{0}=1, a_{1}=1-p w, a_{2}=-q-p-p w, a_{3}=p q w$, and $w=-\ln \sqrt{1+x^{* 2}}+\left(1 /\left(1+x^{* 2}\right)\right)$.

(i) If $x^{*}=0$, one has $a_{0}=1>0, a_{1}=-10<0$. According to Hurwitz stability criterion [19], the necessary condition of stable equilibrium point is the same sign of the coefficients of the characteristic equation. Consequently, the equilibrium $S_{0}$ is unstable.

(ii) If $x^{*}= \pm \sqrt{e^{2}-1}$, one has $a_{0}=1, a_{1}=10.5113>0$, and $a_{2}=-16.3587<0$. Similarly, one can obtain that $S_{+}$and $S_{-}$are the unstable equilibrium points.

Now, we will discuss how to design a linear feedback controller such that the unstable equilibrium points are exponentially stable. For this purpose, we add the control terms to the system (2):

$$
\begin{gathered}
\dot{x}=p\left(x+y-x \ln \sqrt{1+x^{2}}\right)+u_{1}, \\
\dot{y}=x-y+z+u_{2}, \\
\dot{z}=-q y+u_{3} .
\end{gathered}
$$

Let $X^{*}=\left(x^{*}, y^{*}, z^{*}\right)$ be any of the three unstable equilibrium points, let $X(t)=(x(t), y(t), z(t))$ be the solution of the system (25), and $\widetilde{X}(t)=(\tilde{x}, \tilde{y}, \widetilde{z})=X(t)-X^{*}$, then the error system is given by

$$
\begin{gathered}
\dot{\tilde{x}}=p\left(\tilde{x}+\tilde{y}-x \ln \sqrt{1+x^{2}}+x^{*} \ln \sqrt{1+x^{* 2}}\right)+u_{1}, \\
\dot{\tilde{y}}=\tilde{x}-\tilde{y}+\widetilde{z}+u_{2}, \\
\dot{\tilde{z}}=-q \tilde{y}+u_{3} .
\end{gathered}
$$

Definition 5. $\forall X(0)=(x(0), y(0), z(0)) \in R^{3}$, if $u_{i}(i=$ $1,2,3)$ is appropriately selected such that

$$
\left|\widetilde{X}\left(t, t_{0}, X_{0}\right)\right| \leq\left|X(0)-X^{*}\right| e^{-\alpha\left(t-t_{0}\right)}
$$

holds $(\alpha>0)$. Then, the control input $u_{i}(i=1,2,3)$ can globally exponentially stabilize the equilibrium point $X^{*}$.
Theorem 6. If the following linear controller is added to the error system (26),

$$
u_{1}=-p \sigma_{x} \tilde{x}, \quad u_{2}=u_{3}=0,
$$

where $\sigma_{x}$ is any parameter given beforehand such that $\sigma_{x}>2$; then the equilibrium point $X^{*}$ is globally exponentially stable.

Proof. The proof is divided into two steps.

(1) We will find the existence of $\epsilon>0$ such that $G_{3}^{+}$is positive definite and $G_{3}^{-}$is negative definite, where

$$
G_{3}^{+} \triangleq\left[\begin{array}{ccc}
\frac{1}{p} & 0 & 0 \\
0 & 1 & -\frac{\epsilon}{2} \\
0 & -\frac{\epsilon}{2} & \frac{1}{q}
\end{array}\right], \quad G_{3}^{-} \triangleq\left[\begin{array}{ccc}
2\left(1-\sigma_{x}\right) & 2 & -\frac{\epsilon}{2} \\
2 & \epsilon q-2 & \frac{\epsilon}{2} \\
-\frac{\epsilon}{2} & \frac{\epsilon}{2} & -\epsilon
\end{array}\right] .
$$

It is easy to obtain that $G_{3}^{+}$is positive definite if $|\epsilon|<2 / \sqrt{q}$. Now we focus on choosing $\epsilon>0$ such that $G_{3}^{-}$is negative definite. By the Schur theorem [18], $G_{3}^{-}$is negative definite if and only if

$$
\begin{gathered}
\epsilon>0, \\
\epsilon q+\frac{\epsilon}{4}-2<0, \\
\left(2\left(\sigma_{x}-1\right)-\frac{\epsilon}{4}\right)\left(2-\epsilon q-\frac{\epsilon}{4}\right)>\left(2-\frac{\epsilon}{4}\right)^{2},
\end{gathered}
$$

that is

$$
\begin{gathered}
\epsilon>0, \\
\epsilon<\frac{8}{4 q+1}, \\
\sigma_{x}>\frac{64-16 \epsilon+\epsilon^{2}}{64-32 \epsilon q-8 \epsilon}+1+\frac{\epsilon}{8} .
\end{gathered}
$$

Obviously, $G_{3}^{-}$is negative definite if $0<\epsilon \ll 1, \sigma_{x}>2$.

(2) We construct a positive definite and radially unbounded Lyapunov function to prove the stability of closed-loop systems (26) with controller (28) which is written as

$$
\begin{aligned}
V_{1}\left(\widetilde{X}\left(t, t_{0}, \widetilde{X}_{0}\right)\right) & =\frac{1}{p} \widetilde{x}^{2}+\widetilde{y}^{2}+\frac{1}{q} \widetilde{z}^{2}-\epsilon \widetilde{y} \widetilde{z} \\
& =\left[\begin{array}{c}
\tilde{x} \\
\tilde{y} \\
\widetilde{z}
\end{array}\right]^{T} G_{3}^{+}\left[\begin{array}{c}
\tilde{x} \\
\tilde{y} \\
\widetilde{z}
\end{array}\right] .
\end{aligned}
$$

Suppose $\lambda_{m}\left(G_{3}^{+}\right)$and $\lambda_{M}\left(G_{3}^{+}\right)$are minimum and maximum eigenvalues of the positive definite matrix $G_{3}^{+}$, respectively. Then, we have

$$
\lambda_{m}\left(G_{3}^{+}\right)\left(\tilde{x}^{2}+\tilde{y}^{2}+\widetilde{z}^{2}\right) \leq V_{1} \leq \lambda_{M}\left(G_{3}^{+}\right)\left(\tilde{x}^{2}+\tilde{y}^{2}+\widetilde{z}^{2}\right) .
$$


Let $f(\tilde{x})=x \ln \sqrt{1+x^{2}}-x^{*} \ln \sqrt{1+x^{* 2}}$. Obviously, $x \ln \sqrt{1+x^{2}}$ is a monotonically increasing odd function. Then,

(i) if $\tilde{x} \geq 0$, then $f(\widetilde{x}) \geq 0$ and

$$
0 \leq \tilde{x} f(\tilde{x}) \leq+\infty,
$$

(ii) if $\tilde{x} \leq 0$, then $f(\tilde{x}) \leq 0$ and

$$
0 \leq \tilde{x} f(\tilde{x}) \leq+\infty .
$$

Thus

$$
0 \leq \tilde{x} f(\tilde{x}) \leq+\infty, \quad \forall \tilde{x} \in R
$$

Differentiating $V_{1}$ with respect to time yields

$$
\begin{aligned}
\left.\frac{d V_{1}}{d t}\right|_{(26)} & =\frac{2}{p} \tilde{x} \dot{\tilde{x}}+2 \tilde{y} \dot{\bar{y}}+\frac{2}{q} \tilde{z} \dot{\tilde{z}}-\epsilon \dot{\tilde{y}} \tilde{z}-\epsilon \tilde{y} \dot{z} \\
& =\left[\begin{array}{c}
\tilde{x} \\
\tilde{y} \\
\tilde{z}
\end{array}\right]^{T} G_{3}^{-}\left[\begin{array}{c}
\tilde{x} \\
\tilde{y} \\
\tilde{z}
\end{array}\right]-2 \tilde{x} f(\tilde{x}) \\
& \leq \mu_{M}\left(G_{3}^{-}\right)\left(\tilde{x}^{2}+\tilde{y}^{2}+\tilde{z}^{2}\right) \\
& =\mu_{M}\left(G_{3}^{-}\right) \frac{\lambda_{M}\left(G_{3}^{+}\right)}{\lambda_{M}\left(G_{3}^{+}\right)}\left(\tilde{x}^{2}+\tilde{y}^{2}+\widetilde{z}^{2}\right) \leq \frac{\mu_{M}\left(G_{3}^{-}\right)}{\lambda_{M}\left(G_{3}^{+}\right)} V_{1},
\end{aligned}
$$

where $\mu_{M}\left(G_{3}^{-}\right)$is the maximum eigenvalues of the negative definite matrix $G_{3}^{-}$. Then,

$$
\begin{aligned}
V_{1}\left(\widetilde{X}\left(t, t_{0}, \widetilde{X}_{0}\right)\right) \leq & V_{1}\left(\widetilde{X}_{0}\right) e^{\left(\mu_{M}\left(G_{3}\right) / \lambda_{M}\left(G_{3}^{+}\right)\right)\left(t-t_{0}\right)}, \\
\widetilde{x}^{2}(t)+\widetilde{y}^{2}(t)+\widetilde{z}^{2}(t) & \leq \frac{V_{1}\left(\widetilde{X}\left(t, t_{0}, \widetilde{X}_{0}\right)\right)}{\lambda_{m}\left(G_{3}^{+}\right)} \\
& \leq \frac{V_{1}\left(\widetilde{X}_{0}\right)}{\lambda_{m}\left(G_{3}^{+}\right)} e^{\left(\mu_{M}\left(G_{3}^{-}\right) / \lambda_{M}\left(G_{3}^{+}\right)\right)\left(t-t_{0}\right)} .
\end{aligned}
$$

Hence, $\tilde{x}^{2}(t), \tilde{y}^{2}(t)$, and $\widetilde{z}^{2}(t)$ converge to zero exponentially. According to Definition 5 , the equilibrium point $X^{*}$ is globally exponentially stable. The proof is complete.

Remark 7. A constructive method to stabilize the unstable equilibrium points is proposed in this section, matrices $G_{3}^{+}, G_{3}^{-}$, and Lyapunov function candidate $V_{1}$ are given. Then, a linear controller is obtained to solve the problem. Comparing with nonlinear controller, linear controller is easy to implement in reality.

\section{Globally Exponential Synchronization of Two Chua's Systems}

In this section, the globally exponential synchronization of two Chua's systems will be discussed. The drive system is given by

$$
\begin{gathered}
\dot{x}_{d}=p\left(x_{d}+y_{d}-x_{d} \ln \sqrt{1+x_{d}^{2}}\right), \\
\dot{y}_{d}=x_{d}-y_{d}+z_{d}, \\
\dot{z}_{d}=-q y_{d},
\end{gathered}
$$

and the response system is described as follows:

$$
\begin{gathered}
\dot{x}_{r}=p\left(x_{r}+y_{r}-x_{r} \ln \sqrt{1+x_{r}^{2}}\right)+u_{1}, \\
\dot{y}_{r}=x_{r}-y_{r}+z_{r}+u_{2}, \\
\dot{z}_{r}=-q y_{r}+u_{3},
\end{gathered}
$$

where the subscripts $d$ and $r$ denote the drive and response systems and $u_{i}(i=1,2,3)$ is feedback control input which satisfies $u_{i}(0,0,0)=0$. that

Let $e_{x}=x_{r}-x_{d}, e_{y}=y_{r}-y_{d}$, and $e_{z}=z_{r}-z_{d}$; one obtains

$$
\begin{gathered}
\dot{e}_{x}=p\left(e_{x}+e_{y}-x_{r} \ln \sqrt{1+x_{r}^{2}}+x_{d} \ln \sqrt{1+x_{d}^{2}}\right)+u_{1}, \\
\dot{e}_{y}=e_{x}-e_{y}+e_{z}+u_{2}, \\
\dot{e}_{z}=-q e_{y}+u_{3} .
\end{gathered}
$$

Theorem 8. If the following controller is added to the error system (41),

$$
u_{1}=-p \delta_{x} \tilde{x}, \quad u_{2}=u_{3}=0,
$$

where $\delta_{x}$ is any parameter given beforehand with $\delta_{x}>2$; then the zero solution of (41) is globally exponentially stable and the systems (39) and (40) are globally exponentially synchronized.

Proof. Since the proof of this theorem is parallel to that of Theorem 6, we omit it here.

If the parameters $p$ and $q$ in the drive system are uncertain, one can construct the following controlled response system:

$$
\begin{gathered}
\dot{x}_{r}=\widehat{p}\left(x_{r}+y_{r}-x_{r} \ln \sqrt{1+x_{r}^{2}}\right)+u_{1}, \\
\dot{y}_{r}=x_{r}-y_{r}+z_{r}+u_{2}, \\
\dot{z}_{r}=-\widehat{q} y_{r}+u_{3},
\end{gathered}
$$

where $\widehat{p}$ and $\hat{q}$ are the estimates of the uncertain parameters $p$ and $q$, respectively. Let $e_{x}=x_{r}-x_{d}, e_{y}=y_{r}-y_{d}, e_{z}=z_{r}-z_{d}$, 
$\widetilde{p}=\widehat{p}-p$, and $\widetilde{q}=\widehat{q}-q$, then, the error system of (39) and (43) is given by

$$
\begin{gathered}
\dot{e}_{x}=\widehat{p}\left(e_{x}+e_{y}-x_{r} \ln \sqrt{1+x_{r}^{2}}+x_{d} \ln \sqrt{1+x_{d}^{2}}\right) \\
+\widetilde{p}\left(x_{d}+y_{d}-x_{d} \ln \sqrt{1+x_{d}^{2}}\right)+u_{1}, \\
\dot{e}_{y}=e_{x}-e_{y}+e_{z}+u_{2}, \\
\dot{e}_{z}=-\widehat{q} e_{y}-\tilde{q} y_{d}+u_{3} .
\end{gathered}
$$

Theorem 9. If the following adaptive controller is added to the error system (44),

$$
\begin{gathered}
u_{1}=-\widehat{p}\left(e_{x}+e_{y}-x_{r} \ln \sqrt{1+x_{r}^{2}}+x_{d} \ln \sqrt{1+x_{d}^{2}}\right)-e_{x}, \\
u_{2}=-e_{x}-e_{z}, \\
u_{3}=\widehat{q} e_{y}-e_{z}, \\
\dot{\hat{p}}=\dot{\tilde{p}}=-e_{x}\left(x_{d}+y_{d}-x_{d} \ln \sqrt{1+x_{d}^{2}}\right), \\
\dot{\hat{q}}=\dot{\tilde{q}}=e_{z} y_{d},
\end{gathered}
$$

then, we have the following.

(1) The equilibrium points $\left(\tilde{p}=0, \tilde{q}=0, e_{x}=0, e_{y}=0\right.$, $e_{z}=0$ ) of the system (44) with adaptive control law (45) are globally stable; in addition, $\lim _{t \rightarrow \infty} e_{x}=0$, $\lim _{t \rightarrow \infty} e_{y}=0$, and $\lim _{t \rightarrow \infty} e_{z}=0$. And thus, the two systems (39) and (44) are globally synchronized.

(2) The parameter estimates $\hat{p}$ and $\hat{q}$ will, respectively, converge to $p$ and $q$ as $t$ tends to infinity.

Proof. The proof contains two steps.

Firstly, a Lyapunov function candidate is constructed as follows:

$$
V_{2}=\frac{1}{2}\left(e_{x}^{2}+e_{y}^{2}+e_{z}^{2}+\widetilde{p}^{2}+\widetilde{q}^{2}\right) .
$$

Then, one has

$$
\begin{aligned}
\left.\frac{d V_{2}}{d t}\right|_{(44)}= & e_{x} \dot{e}_{x}+e_{y} \dot{e}_{y}+e_{z} \dot{e}_{z}+\tilde{p} \dot{\tilde{p}}+\tilde{q} \dot{\tilde{q}} \\
= & e_{x}\left(\hat { p } \left(e_{x}+e_{y}-x_{r} \ln \sqrt{1+x_{r}^{2}}\right.\right. \\
& \left.\left.+x_{d} \ln \sqrt{1+x_{d}^{2}}\right)+u_{1}\right) \\
& +e_{y}\left(e_{x}-e_{y}+e_{z}+u_{2}\right) \\
& +e_{z}\left(-\hat{q} e_{y}+u_{3}\right)-\tilde{q}\left(e_{z} y_{d}-\dot{\tilde{q}}\right) \\
& +\widetilde{p}\left(e_{x}\left(x_{d}+y_{d}-x_{d} \ln \sqrt{1+x_{d}^{2}}\right)+\dot{\tilde{p}}\right) \\
= & -e_{x}^{2}-e_{y}^{2}-e_{z}^{2} \leq 0 .
\end{aligned}
$$




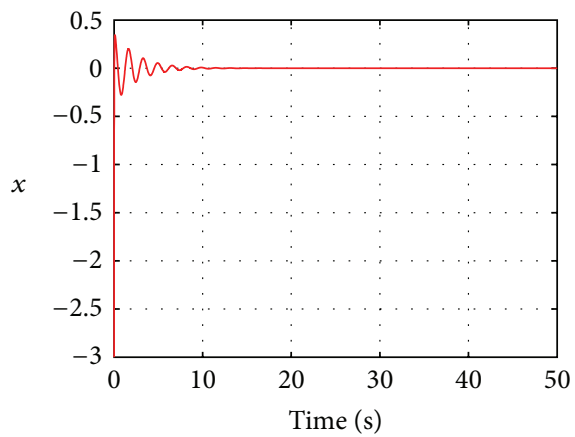

(a)

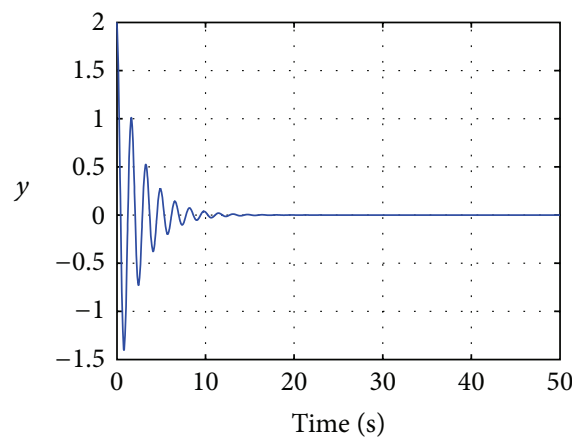

(b)

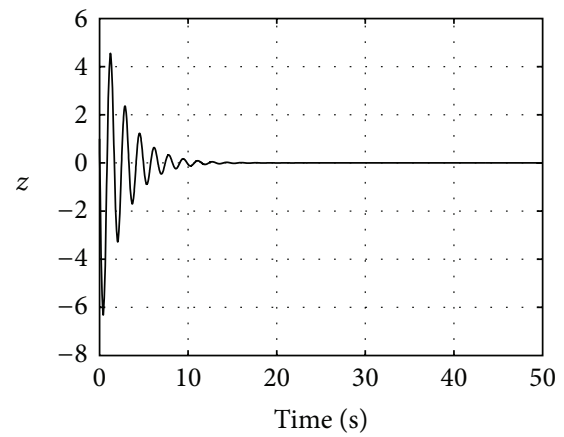

(c)

FIGURE 2: The asymptotic stability of $S_{0}$ with controller (28).

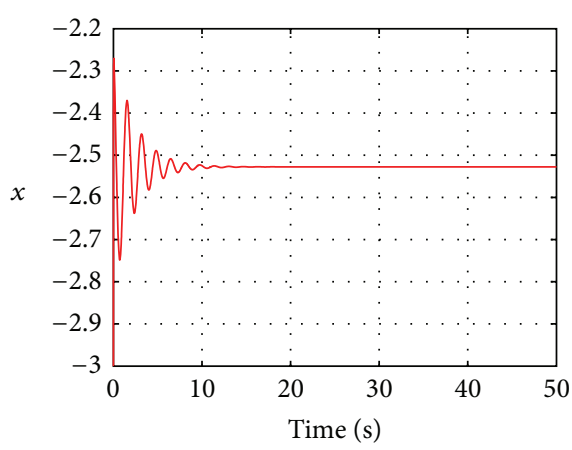

(a)

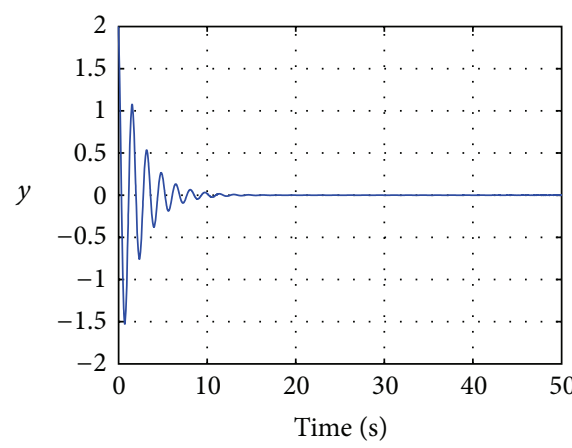

(b)

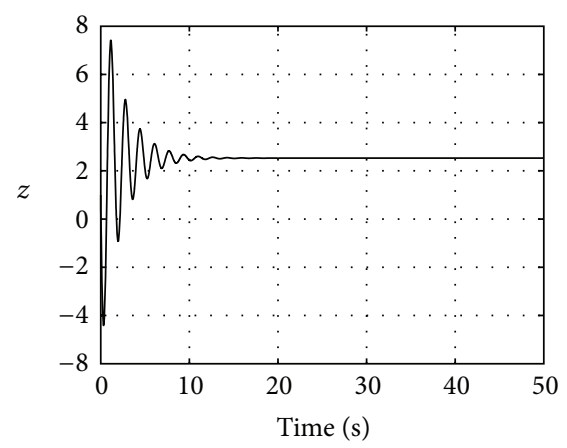

(c)

FIgURE 3: The asymptotic stability of $S_{-}$with controller (28).

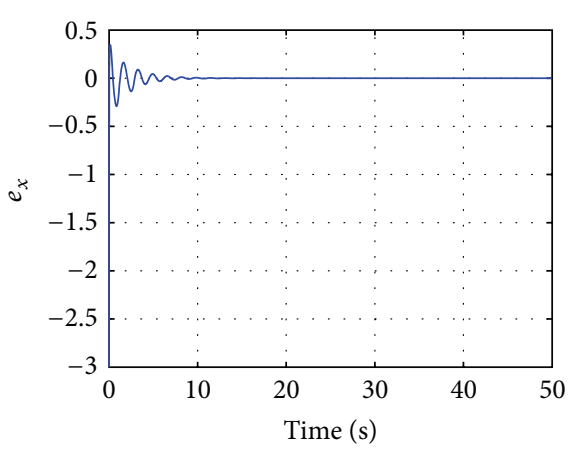

(a)

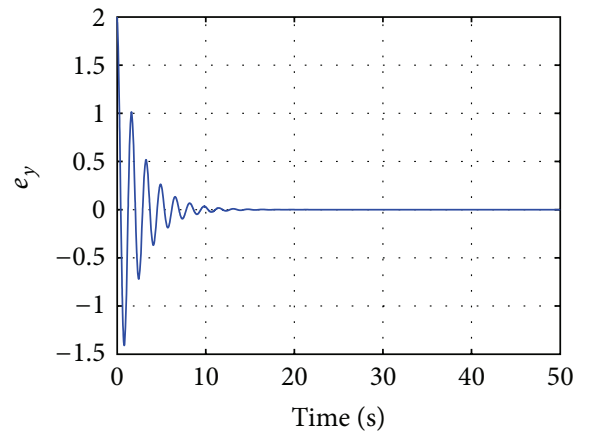

(b)

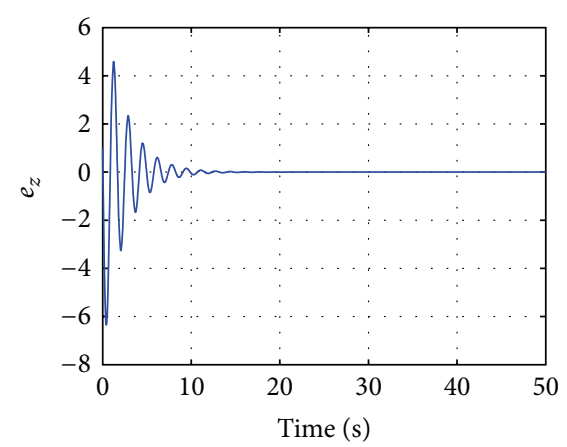

(c)

Figure 4: The synchronization errors of the system (39) and (40) with linear controller (42).

\section{Numerical Simulations}

In this section, several examples of numerical simulations are proposed to illustrate the theoretical results obtained in the previous sections. A fourth-order Runge-Kutta method is used to obtain the simulation results with MATLAB.

Chua's system (2) and the error systems (26), (41), and (44) are considered in this section for the numerical simulations. Let $p=11, q=14.87$, the initial state $x(0)=-3$, $y(0)=2$, and $z(0)=1$. Figure 2 shows the state trajectories of the closed-loop system (26) with linear control input (28); it is easy to see that the equilibrium point $S_{0}$ is asymptotically stable. Similarly, Figure 3 shows that, with the corresponding control input (28), the equilibrium point $S_{-}$is asymptotically stable. Figure 4 shows the synchronous errors of the system (39) and (40) with linear controller (42); it is easy to see that the two systems are globally asymptotically synchronized. When parameters $p$ and $q$ are uncertain, by using adaptive controller (45), the synchronous errors are asymptotically convergent to $e_{x}=0, e_{y}=0, e_{z}=0$ (see Figure 5), 


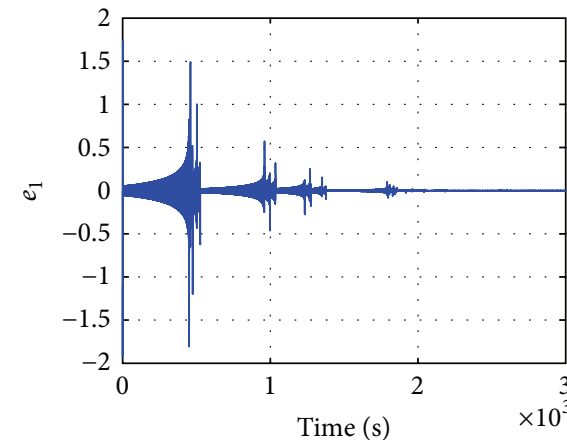

(a)

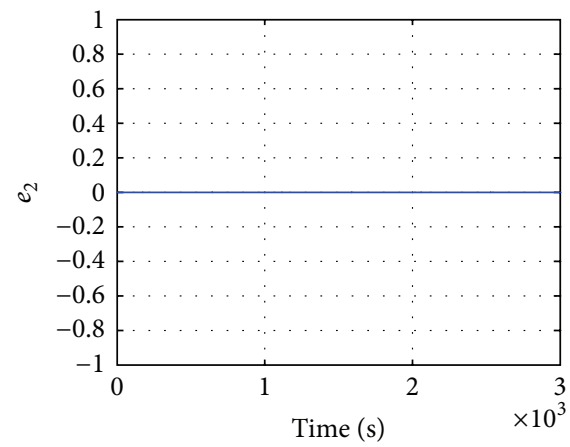

(b)

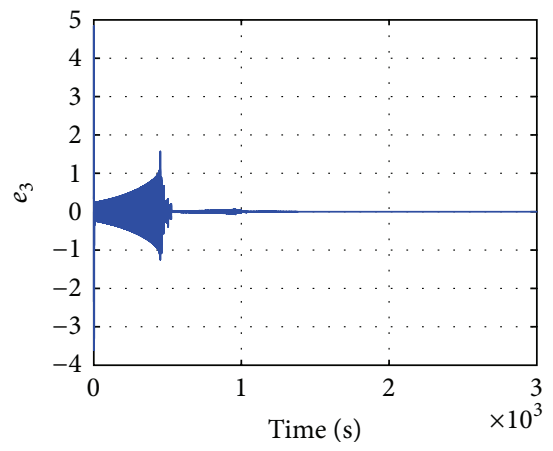

(c)

FIGURE 5: The synchronization errors of the system (39) and (43) with adaptive controller (45).

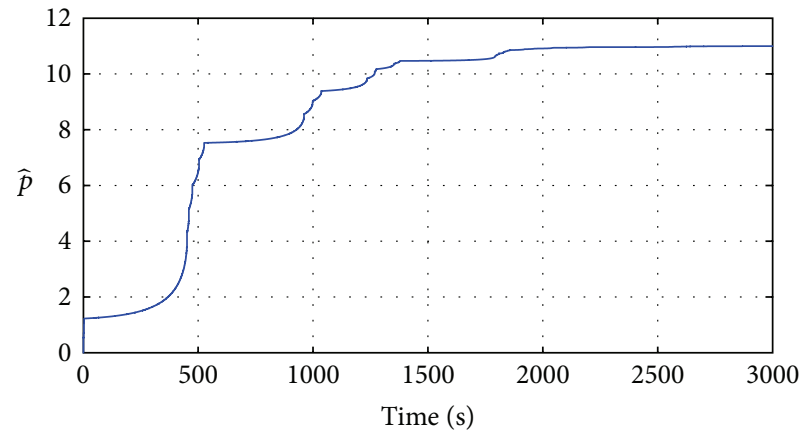

(a)

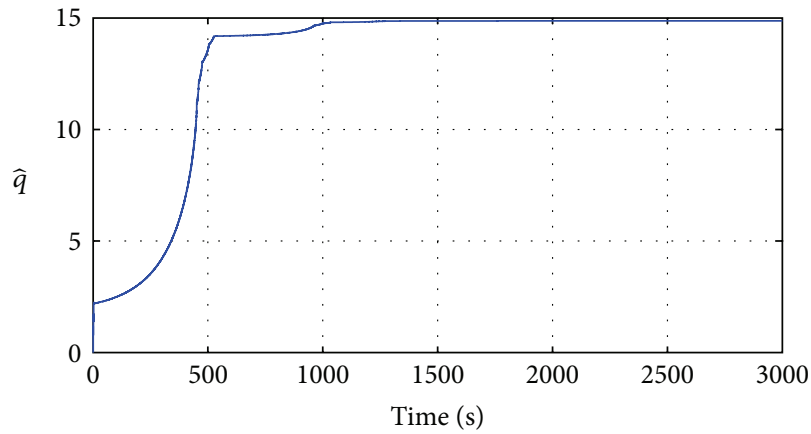

(b)

FIGURE 6: The convergence of parameters $\widehat{p}$ and $\widehat{q}$.

and the estimate values of the uncertain parameters $p$ and $q$ asymptotically converge to the real values $p=11, q=14.87$ (see Figure 6).

\section{Conclusions}

A new smooth Chua's system is constructed, and the chaotic characteristics is confirmed by computing the Lyapunov exponents of the system. A Constructive method is used to prove the existence of globally exponential attractive set and positive invariant set. For the three unstable equilibrium points of the system, a linear controller is designed to achieve globally exponential stability of the equilibrium points. Then, a linear controller and an adaptive controller are, respectively, proposed so that two similar types of smooth Chua's systems are globally synchronized, and the estimate errors of the uncertain parameters converge to zero as $t$ tends to infinity.

\section{Acknowledgments}

The authors want to express their sincere thanks to the editor and the referee for their invaluable comments and suggestions which helped improve the paper greatly. This work was supported by the National Natural Science Foundation of China (50937002 and 51207063) and the Project of the Education Department of Hubei Province (T200910, T201009, and D20132801).

\section{References}

[1] L. O. Chua, "The genesis of Chua's circuit," Archiv fur Elektronik und Ubertragungstechnik, vol. 46, no. 4, pp. 250-257, 1992.

[2] L. P. Shil'nikov, "Chua's circuit: rigorous results and future problems," International Journal of Bifurcation and Chaos, vol. 4, no. 3, pp. 489-519, 1994.

[3] L. O. Chua, C. W. Wu, A. Huang, and G.-Q. Zhong, "A universal circuit for studying and generating chaos I: routes to chaos," IEEE Transactions on Circuits and Systems I, vol. 40, no. 10, pp. 732-744, 1993.

[4] L. O. Chua, "A zoo of strange attractor from the canonical Chua's circuits," in Proceedings of the 35th Midwest Symposium on Circuits and Systems, vol. 2, pp. 916-926, 1992.

[5] Y. F. Wang and J. G. Jiang, "The chaotic phenomena analysis of asymmetric nonlinear Chua's circuit," Systems Engineering and Electronics, vol. 29, no. 12, pp. 2029-2031, 2007.

[6] K. S. Tang, K. F. Man, G. Q. Zhong, and G. Chen, "Generating chaos via $x|x|$," IEEE Transactions on Circuits and Systems I, vol. 48 , no. 5, pp. 636-641, 2001. 
[7] X. Liao, P. Yu, S. Xie, and Y. Fu, "Study on the global property of the smooth Chua's system," International Journal of Bifurcation and Chaos in Applied Sciences and Engineering, vol. 16, no. 10, pp. 2815-2841, 2006.

[8] H. M. Deng, T. Li, Q. H. Wang et al., "Shaped Chua’s chaotic system and its synchronization problem," Systems Engineering and Electronics, vol. 31, no. 3, pp. 638-641, 2009.

[9] G. A. Leonov, A. I. Bunin, and N. Koksch, "Attractor localization of the Lorenz system," Zen and the Art of Motorcycle Maintenance, vol. 67, no. 12, pp. 649-656, 1987.

[10] F. Zhou, Z. Y. Wang, G. P. Zhou, and F. X. Zhen, "Synchronization of two unsmooth Chua's circuits," Mathematica Applicata, vol. 25, no. 2, pp. 382-388, 2012.

[11] X. X. Liao, H. G. Luo, Y. L. Fu et al., "Positive invariant set and the globally exponentially attractive set of Lorenz system group," Science in China-E, vol. 37, no. 6, pp. 757-769, 2007.

[12] X. X. Liao, "New results for globally attractive set and positive invariant set of Lorenz system and application of chaos control and synchronization," Science in China-E, vol. 34, no. 12, pp. 1404-1419, 2004.

[13] J. G. Jian, X. L. Deng, and J. F. Wang, "New results of globally exponentially attractive set and synchronization controlling of the Qi chaotic system," Advances in N Eural Networks-ISNN, pp. 643-650, 2010.

[14] F. Q. Wang and C. X. Liu, "A new criterion for chaos and hyperchaos synchronization using linear feedback control," Physics Letters A, vol. 360, no. 2, pp. 274-278, 2006.

[15] H. R. Koofigar, F. Sheikholeslam, and S. Hosseinnia, "Robust adaptive synchronization for a general class of uncertain chaotic systems with application to Chuas circuit," Chaos, vol. 21, no. 4, Article ID 043134, 2011.

[16] H. G. Zhang, W. Huang, Z. L. Wang et al., "Adaptive synchronization between two different chaotic systems with unknown parameters," Physics Letters A, vol. 350, no. 5-6, pp. 363-366, 2006.

[17] F. Zhou, Z. Y. Wang, and G. P. Zhou, "Adaptive chronization of some modified smooth Chua's circuit," Journal of Nanjing University of Information Science and Techology, vol. 4, no. 2, pp. 186-189, 2012.

[18] X. X. Liao, Theory Methods and Application of Stability, Huazhong University of Science and Technology Press, 2nd edition, 2010.

[19] C. L. Phillips and J. Parr, Feedback Control Systems, Prentice Hall, 5th edition, 2010.

[20] M. Kristic, I. Kanellakopoulos, and P. Kokotovic, Nonlinear and Adaptive Control Design, John Wiley \& Sons, New York, NY, USA, 1995.

[21] L. Liu, Z. Chen, and J. Huang, "Parameter convergence and minimal internal model with an adaptive output regulation problem," Automatica, vol. 45, no. 5, pp. 1306-1311, 2009.

[22] S. Tong and Y. Li, "Observer-based fuzzy adaptive control for strict-feedback nonlinear systems," Fuzzy Sets and Systems, vol. 160, no. 12, pp. 1749-1764, 2009.

[23] G. Zhou and C. Wang, "Deterministic learning from control of nonlinear systems with disturbances," Progress in Natural Science, vol. 19, no. 8, pp. 1011-1019, 2009. 


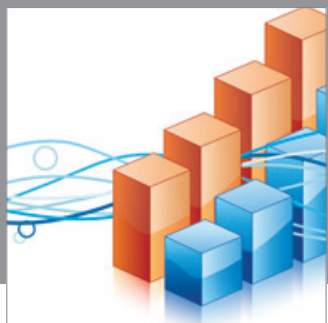

Advances in

Operations Research

mansans

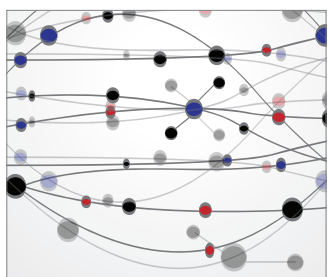

The Scientific World Journal
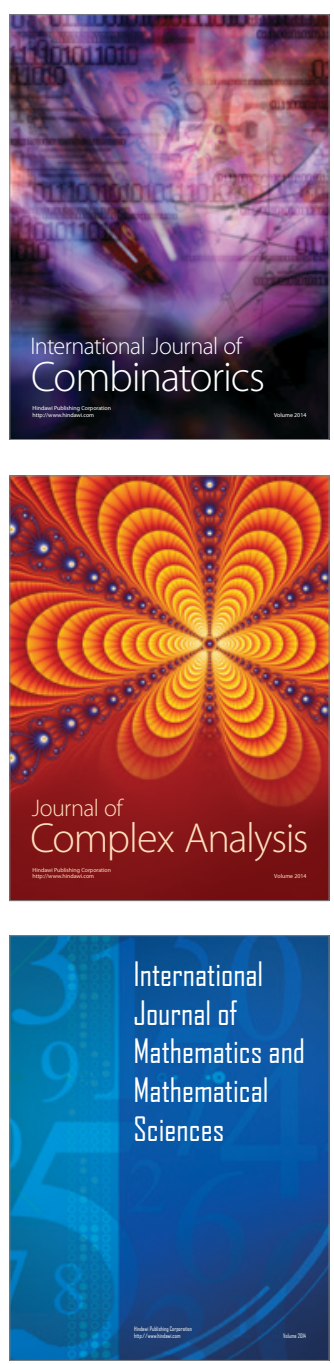
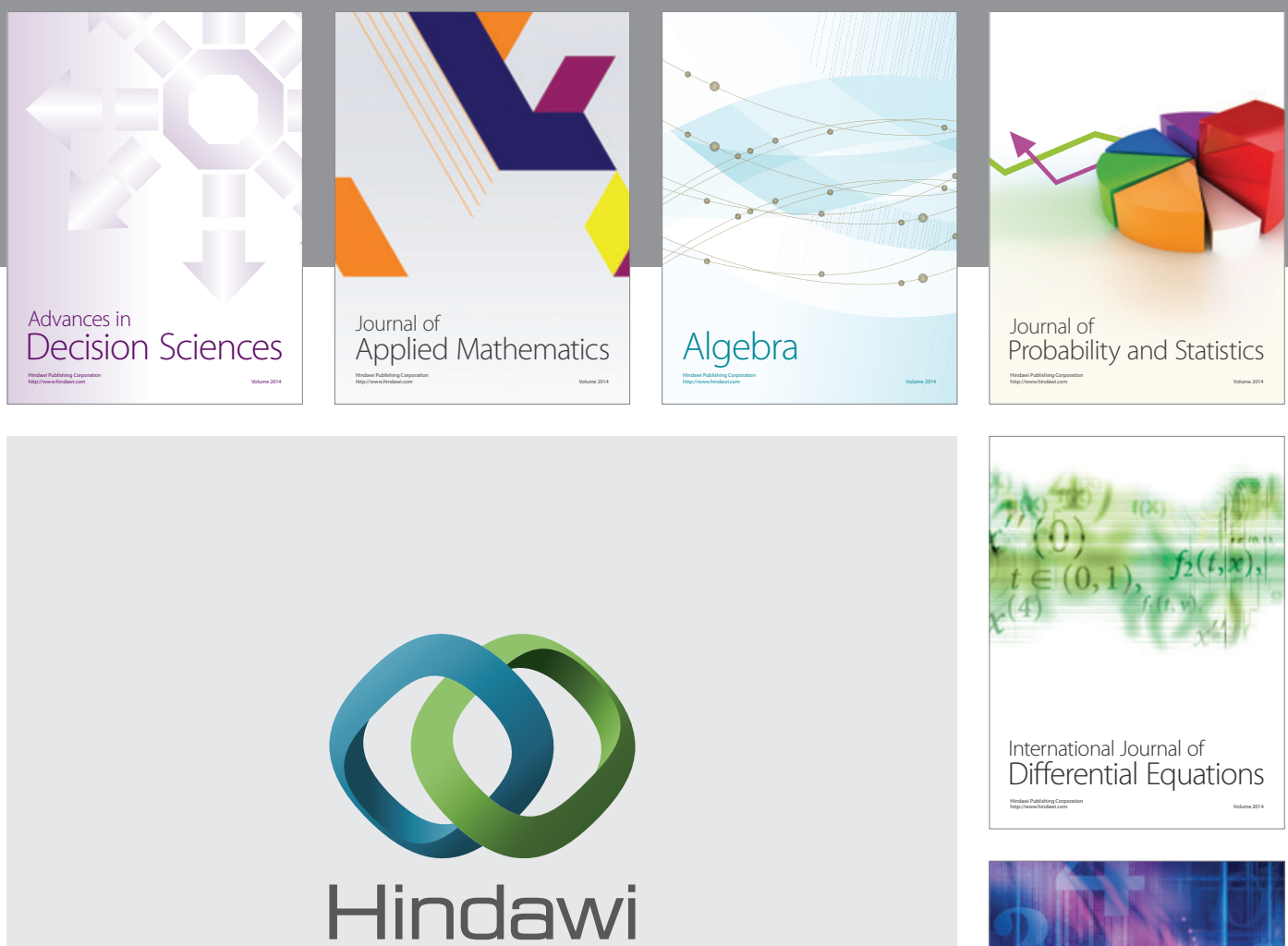

Submit your manuscripts at http://www.hindawi.com
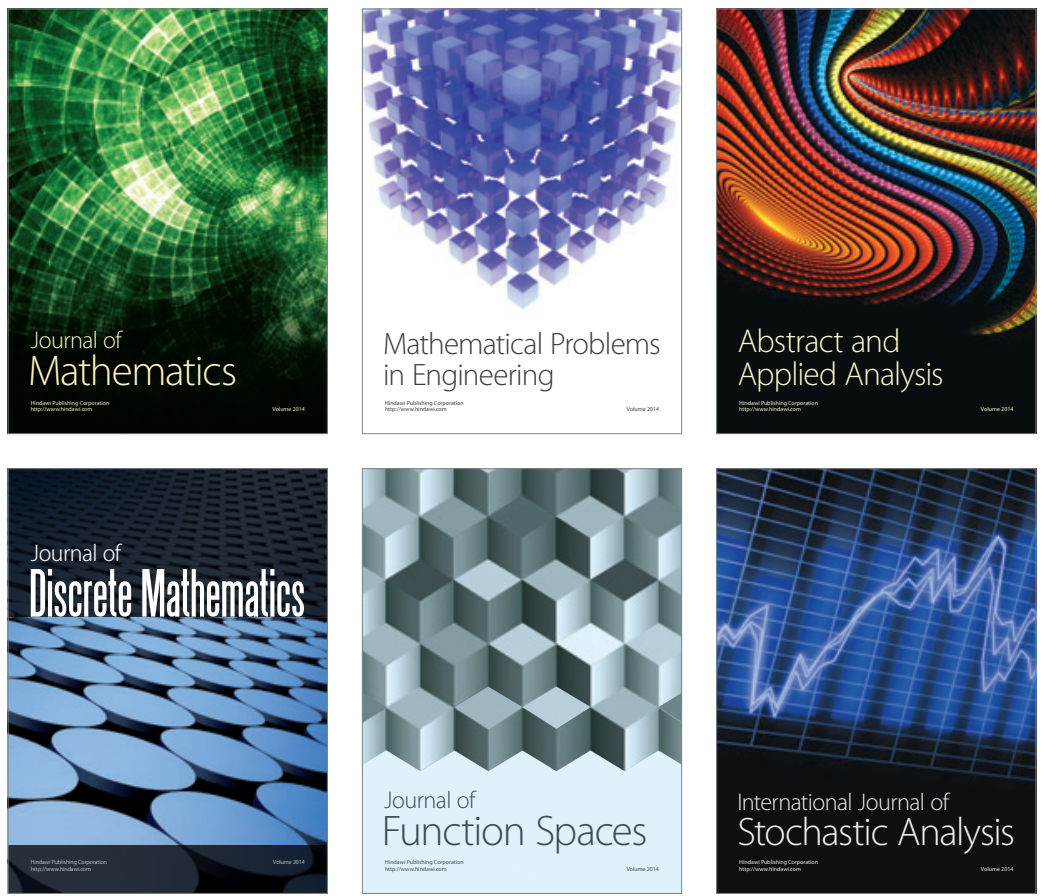

Journal of

Function Spaces

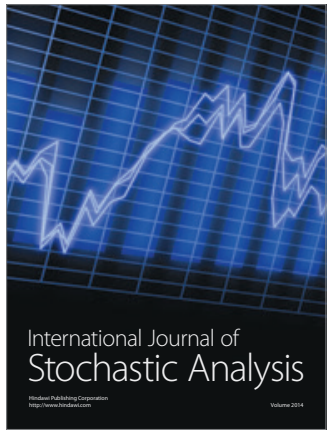

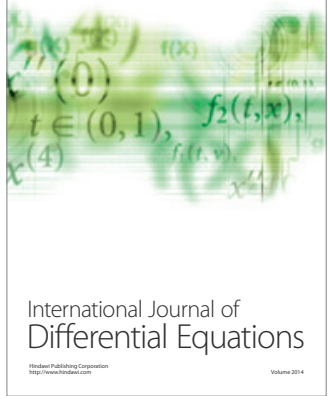
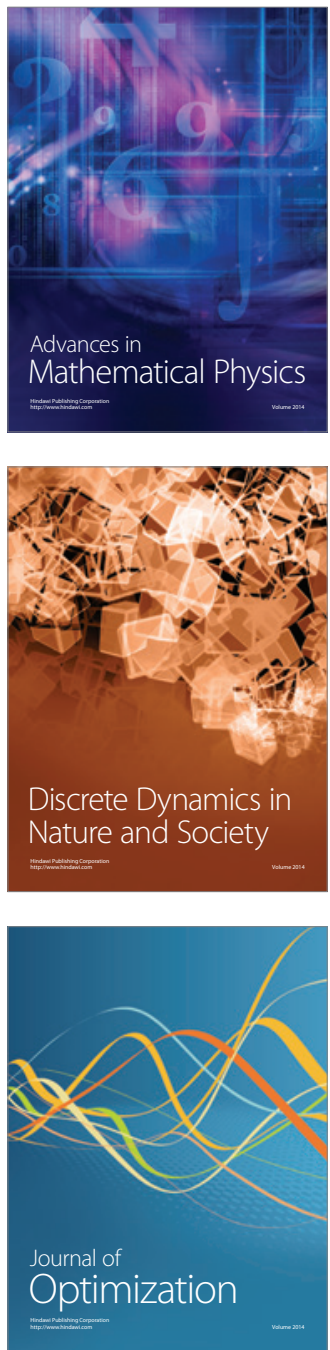